\title{
Associação entre o índice de massa corporal e a coordenação motora em crianças
}

CDD. 20.ed. 152.3

613.7

616.398
Maria Mafalda MELO* Vitor Pires LOPES S******$^{* *}$

\section{Resumo}

0 propósito do estudo foi analisar a associação entre o índice de massa corporal (IMC) e a coordenação motora (CM). Foram avaliadas 794 crianças (seis a nove anos) de ambos sexos na CM, com a bateria de testes KTK. O IMC foi calculado a partir das medidas de peso e estatura [Peso(kg)/Estatura $\left.\left(\mathrm{cm}^{2}\right)\right]$. Quer nas meninas $\left(\chi^{2}=93,96 ; p<0,001\right)$ quer nos meninos $\left(\chi^{2}=46,98 ; p<0,001\right)$ ocorreram diferenças significativas na CM entre os três grupos do IMC (normoponderais, sobrepeso, obesos). Os normoponderais de ambos os sexos obtiveram melhores resultados do que os sujeitos com sobrepeso e estes obtiveram melhores resultados do que os obesos. A CM está moderada e negativamente associada com o IMC e a associação aumenta durante a infância $(-0,16$ a $-0,50)$. As crianças com sobrepeso e obesas de ambos os sexos apresentaram menores níveis de $\mathrm{CM}$ do que as crianças normoponderais.

Palavras-chave: Obesidade; Desempenho psicomotor; Atividade física.

\section{Introdução}

Os níveis de obesidade têm aumentado globalmente. A Organização Mundial de Saúde estimou que em 2005 pelo menos 20 milhões de crianças com idade inferior a cinco anos tinham sobrepeso/ obesidade $^{1}$. Na Europa a prevalência do sobrepeso e da obesidade na adolescência estava entre três e 35\% nas crianças com 13 anos de idade e entre cinco e $28 \%$ nos adolescentes com 15 anos de idade ${ }^{2}$. Em Portugal, $31,5 \%$ das crianças entre os sete e os nove anos de idade são obesos ou têm sobrepeso ${ }^{3}$.

As principais causas da obesidade são, para além de fatores genéticos ${ }^{4}$, o elevado aporte energético contido na dieta e o sedentarismo 5 . A atividade física (AF) é um fator primordial na prevenção do sobrepeso e obesidade. Ela desempenha um papel fundamental no balanço energético e portanto no controlo ponderal. Na generalidade o excesso de peso está associado a níveis baixos de $\mathrm{AF}^{6}$. Apesar de vários estudos terem mostrado que as crianças e os adolescentes obesos são menos ativos do que os não obesos, as relações entre a $\mathrm{AF}$, o sedentarismo (por exemplo ver televisão) e o estatuto ponderal na infância e juventude não estão ainda bem esclarecidas? .

A AF é um comportamento complexo que está dependente e/ou correlacionado com vários fatores.
A generalidade das investigaçôes tem-se debruçado sobre a influência de variáveis psicológicas e do envolvimento nos níveis de AF das crianças. Em algumas revisões da literatura ${ }^{8-9}$, constatou-se que num conjunto vasto de variáveis, apesar de estarem significativamente associadas com a $\mathrm{AF}$, o valor das correlaçóes era baixo, explicando uma percentagem de variância pequena da AF, $18 \%$ para os rapazes entre os 10 e os 12 anos de idade ${ }^{10}$. Um número reduzido de estudos debruçou-se sobre o papel da competência motora nos níveis de AF habitual. Alguns estudos mostraram que as crianças com maiores níveis de proficiência em habilidades motoras eram mais ativas do que as crianças com baixos níveis de proficiência ${ }^{11-16}$, estas por seu lado apresentavam uma motivação inferior para a $\mathrm{AF}^{17}$.

A generalidade da literatura em $\mathrm{AF}$ não tem dado importância ao desenvolvimento da competência motora e ao seu papel na promoção de uma vida fisicamente ativa. A investigação tem-se focado na medição da AF em crianças sem a compreensão que aprender a mover-se é um aspecto fundamental na AF. Se as crianças de forma proficiente não correm, saltam, agarram, lançam, trepam, etc. terão
*Faculdade de Desporto, Universidade do Porto - Portugal. ${ }^{*}$ Centro de Investigação em Desporto, Saúde e Desenvolvimento Humano, Universidade da

Beira Interior - Portugal. ***Instituto Politécnico de Bragança - Portugal. 
oportunidades limitadas para se envolverem em atividades físicas dado que não terão um reportório motor suficiente. As crianças necessitam possuir um largo reportório de habilidades motoras para participar em diferentes atividades físicas. Com um largo reportório de habilidades motoras, as crianças terão maior oportunidade de encontrar atividades físicas que executem bem e gostem. Consideramos, portanto, que a competência motora é o fator central no envolvimento em AF. É a capacidade para se mover de forma coordenada em diferentes

\section{Método}

\section{Amostra}

A amostra é constituída por 794 crianças de ambos os sexos (meninas: $\mathrm{n}=398$; meninos: $\mathrm{n}=396$ ) com idades compreendidas entre os seis e os nove anos. Todas as crianças pertenciam ao agrupamento de escolas do Ovar no distrito de Aveiro (região centro de Portugal). Não houve processo aleatório de amostragem, procurou incluir-se todas as crianças do referido agrupamento de escolas. A existência de doença ou deficiência que impedisse a realização dos testes e a eventual recusa em participar foram os critérios de exclusão utilizados.

Os pais e as crianças foram informados dos procedimentos do estudo e deram o seu consentimento por escrito, sendo o estudo aprovado pela comissão de ética do Instituto Politécnico de Bragança.

\section{Procedimentos}

Para a obtenção dos valores do peso, foi utilizada uma balança digital, tendo as crianças vestuário mínimo, isto é, calção e camiseta. A estatura foi avaliada com as crianças descalças entre o vértex e o plano de referência do solo utilizando um estadiômetro.

O Índice de Massa corporal (IMC) foi calculado através da fórmula: Peso $(\mathrm{kg}) /$ Estatura $\left(\mathrm{cm}^{2}\right)$. Foram utilizados os critérios da Internacional Task Force for the Study of Obesity (IOTF $)^{18}$ para classificar os sujeitos da amostra quanto à obesidade e sobrepeso.

O teste de coordenação motora para crianças [Körperkoordinationstest für Kinder (KTK) $]^{19}$ foi o instrumento utilizado para a avaliar a coordenação motora. O KTK é composto por quatro provas:

- Equilíbrio em marcha para atrás: a tarefa a executar consiste em caminhar para trás sobre três situações e tarefas que representa a trave mestra para os sujeitos serem ativos e por consequência não desenvolverem sobrepeso e obesidade.

O objetivo desta investigação é analisar a associação entre a coordenação motora e o índice de massa corporal (IMC) em crianças de ambos os sexos com idades comprendidas entre os seis e os nove anos de idade, tendo como hipótese que as crianças de ambos os sexos com sobrepeso e obesidade apresentam níveis de coordenação motora inferiores às crianças normoponderais.

traves de madeira com três metros de comprimento, $3 \mathrm{~cm}$ de altura e com uma largura de $6 \mathrm{~cm}, 4,5 \mathrm{~cm}$ e $3 \mathrm{~cm}$ respectivamente. São válidas três tentativas por cada trave. Durante o deslocamento (passos) não é permitido tocar com os pés no chão. Os deslocamentos realizam-se por ordem decrescente de largura das traves, sendo contabilizados o número de passos.

- Saltos monopedais: a tarefa consiste em saltar a um pé (primeiro o pé preferido e depois o outro) por cima de uma ou mais placas de $5 \mathrm{~cm}$ de altura. A criança deve começar o salto de acordo com a altura recomendada para a idade. Caso o aluno não obtenha êxito na altura inicial de prova deverá recuar $5 \mathrm{~cm}$ na altura até obter êxito. A recepção deverá ser feita com o mesmo pé com que iniciou o salto. São permitidas três tentativas em cada altura a saltar para executar o salto, sendo atribuídos 3 pontos se o êxito for obtido na primeira tentativa; 2 pontos se o êxito for obtido na segunda tentativa; 1 ponto se o êxito for obtido na terceira tentativa e zero pontos no insucesso.

- Saltos laterais: a tarefa consiste em saltar lateralmente, com os pés unidos, durante 15 segundos tão rapidamente quanto possível de um lado para o outro de um obstáculo sem a tocar e dentro duma área delimitada. São contabilizados o número de saltos.

- Transposição lateral: a tarefa consiste na transposição lateral de duas plataformas durante 20 segundos. São permitidas duas tentativas válidas. O sujeito coloca-se sobre uma das plataformas, por exemplo a do seu lado direito; ao sinal de partida pega, com as duas mãos, na plataforma que se encontra ao seu lado esquerdo colocando-a ao seu lado direito; de seguida passa o seu corpo para essa plataforma e repete a sequência. São contabilizados o número de transposições. 
Todas as provas visam a caracterização de facetas de coordenação corporal total e o domínio corporal. $\mathrm{O}$ resultado de cada item foi comparado com os valores normativos fornecidos pelo manual, sendo atribuído a cada item um quociente. Do somatório dos quatro quocientes resulta o quociente motor (QM) que é um indicador ajustado à idade e sexo e que permite classificar as crianças segundo o seu nível de desenvolvimento coordenativo: perturbaçôes da coordenação; insuficiência coordenativa; coordenação normal; coordenação boa; coordenação muito boa.

\section{Análise estatística}

A amostra foi estratificada por idade de sexo. Sendo incluídas em cada grupo etário as crianças cuja

\section{Resultados}

$\mathrm{Na}$ TABELA 1 consta a estatística descritiva (média e desvio-padrão) das variáveis avaliadas, por sexo e por idade.

$\mathrm{O}$ valores do QM mostram, de forma clara, uma tendência para os meninos apresentarem, em todas as idades, melhor desempenho coordenativo do que as meninas. Os valores médios do QM são em geral baixos sendo, de acordo com o manual da bateria KTK, indicador de insuficiência coordenativa. idade estivesse compreendida entre o valor inteiro e nove décimas da respectiva idade decimal, por exemplo, uma criança seria considerada no grupo seis anos caso tivesse uma idade entre 6,0 e 6,9 anos decimais. Foi calculada a estatística descritiva (média e desvio-padrão) para as diferentes variáveis. Para testar a normalidade das distribuições foi utilizado o teste de Kolmogorov-Smirnov com a correção de Lilliefors. Dado que quer o QM quer o IMC não apresentavam uma distribuição normal em todos os grupos etários e sexo, foi utilizado o coeficiente de correlação Spearman para analisar a associação entre o IMC e o QM em cada idade e por sexo. O teste de Kruskal-Wallis foi utilizado para analisar as diferenças no QM entre as crianças das diferentes categorias de IMC (normoponderais, com sobrepeso, obesas).

TABELA 1 - Estatística descritiva (média \pm desvio-padrão) para as variáveis avaliadas por sexo e por idade.

\begin{tabular}{cccccc}
\hline \multirow{2}{*}{ Idade (anos) } & $\mathbf{5}$ Meninas \\
\cline { 2 - 6 } & $\mathbf{n}$ & Peso $(\mathbf{k g})$ & Altura $(\mathbf{c m})$ & IMC $\left(\mathbf{k g}^{\circ} \mathbf{m}^{-1}\right)$ & QM (\# pontos) \\
\hline 6 & 44 & $25 \pm 6$ & $123,9 \pm 7,1$ & $16,1 \pm 3,0$ & $77 \pm 12$ \\
7 & 105 & $28 \pm 6$ & $126,8 \pm 6,9$ & $17,3 \pm 2,6$ & $79 \pm 14$ \\
8 & 125 & $32 \pm 8$ & $134,7 \pm 8,3$ & $17,5 \pm 3,2$ & $74 \pm 14$ \\
9 & 124 & $35 \pm 9$ & $138,1 \pm 8,7$ & $18,3 \pm 3,5$ & $70 \pm 14$ \\
\hline 6 & 34 & Meninos & & $78 \pm 14$ \\
7 & 120 & $26 \pm 5$ & $125,5 \pm 5,8$ & $16,40 \pm 2,6$ & $84 \pm 14$ \\
9 & 118 & $32 \pm 8$ & $134,4 \pm 7,8$ & $17,60 \pm 3,2$ & $82 \pm 13$ \\
\hline
\end{tabular}

IMC: Índice de massa corporal; $\mathrm{QM}$ : quociente motor.
Na TABELA 2 são apresentados os valores percentuais quer das meninas quer dos meninos que se encontram nas categorias do IMC de acordo com os valores de corte do IOTF $^{18}$. A prevalência de sobrepeso e de obesidade é de 20 e 9,8\% respetivamente.

$\mathrm{Na}$ TABELA 3 são apresentadas as médias e os desvios-padrão, do quociente motor por categorias do $\mathrm{IMC}^{18}$. 
TABELA 2 - Percentagem de meninas e meninos em cada categoria do IMC de acordo com os valores de corte do $\mathrm{IOTF}^{18}$.

\begin{tabular}{ccccccc}
\hline \multirow{2}{*}{ Idade (anos) } & \multicolumn{3}{c}{ Meninas } & \multicolumn{3}{c}{ Meninos } \\
\cline { 2 - 6 } & Normoponderal & Sobrepeso & Obeso & Normoponderal & Sobrepeso & Obeso \\
\hline 6 & $72,7 \%$ & $15,9 \%$ & $11,4 \%$ & $67,6 \%$ & $23,5 \%$ & $8,8 \%$ \\
7 & $61,9 \%$ & $22,9 \%$ & $15,2 \%$ & $72,5 \%$ & $22,5 \%$ & $5,0 \%$ \\
8 & $68,0 \%$ & $22,4 \%$ & $9,6 \%$ & $74,6 \%$ & $12,7 \%$ & $12,7 \%$ \\
9 & $69,4 \%$ & $21,8 \%$ & $8,9 \%$ & $71,8 \%$ & $20,2 \%$ & $8,1 \%$ \\
\hline
\end{tabular}

TABELA 3 - Média e desvio-padrão dos valores do quociente motor por categoria do IMC $^{18}$.

\begin{tabular}{ccccccc}
\hline \multirow{2}{*}{ Idade (anos) } & \multicolumn{3}{c}{ Meninas } & \multicolumn{3}{c}{ Meninos } \\
\cline { 2 - 7 } & Normoponderal & Sobrepeso & Obeso & Normoponderal & Sobrepeso & Obeso \\
\hline 6 & $79,0 \pm 9,7$ & $77,4 \pm 13,5$ & $59,4 \pm 5,7$ & $79,7 \pm 12,9$ & $80,4 \pm 16,6$ & $58,7 \pm 2,3$ \\
7 & $82,8 \pm 12,9$ & $83,9 \pm 8,1$ & $58,8 \pm 5,7$ & $86,1 \pm 13,1$ & $80,5 \pm 13,0$ & $65,3 \pm 9,6$ \\
8 & $77,1 \pm 12,5$ & $73,3 \pm 10,9$ & $48,8 \pm 8,8$ & $83,2 \pm 13,8$ & $79,6 \pm 10,0$ & $73,9 \pm 4,5$ \\
9 & $74,7 \pm 11,4$ & $66,9 \pm 9,4$ & $44,1 \pm 4,2$ & $78,1 \pm 13,2$ & $71,2 \pm 14,4$ & $61,4 \pm 10,7$ \\
\hline
\end{tabular}

Quer nas meninas $\left(\chi^{2}=93,96 ; \mathrm{p}<0,001\right)$ quer nos meninos $\left(\chi^{2}=46,98 ; \mathrm{p}<0,001\right)$ ocorreram diferenças significativas no QM entre os três grupos do IMC. As crianças normoponderais de ambos os sexos têm melhores resultados do que as que têm sobrepeso e estas, por sua vez, têm melhores resultados do que as obesas (FIGURA 1).
Na TABELA 4 constam os valores do coeficiente de correlação de Spearman entre o IMC e o QM. Todas as correlações são negativas e variam entre -0,16 e -0,50. Os valores de correlação são muitos semelhantes entre as meninas e os meninos, exceto aos nove anos de idade. Os valores de correlação aumentam com a idade até aos nove anos de idade, exceto nos meninos que apresentam um decréscimo entre os oito e os nove anos de idade.

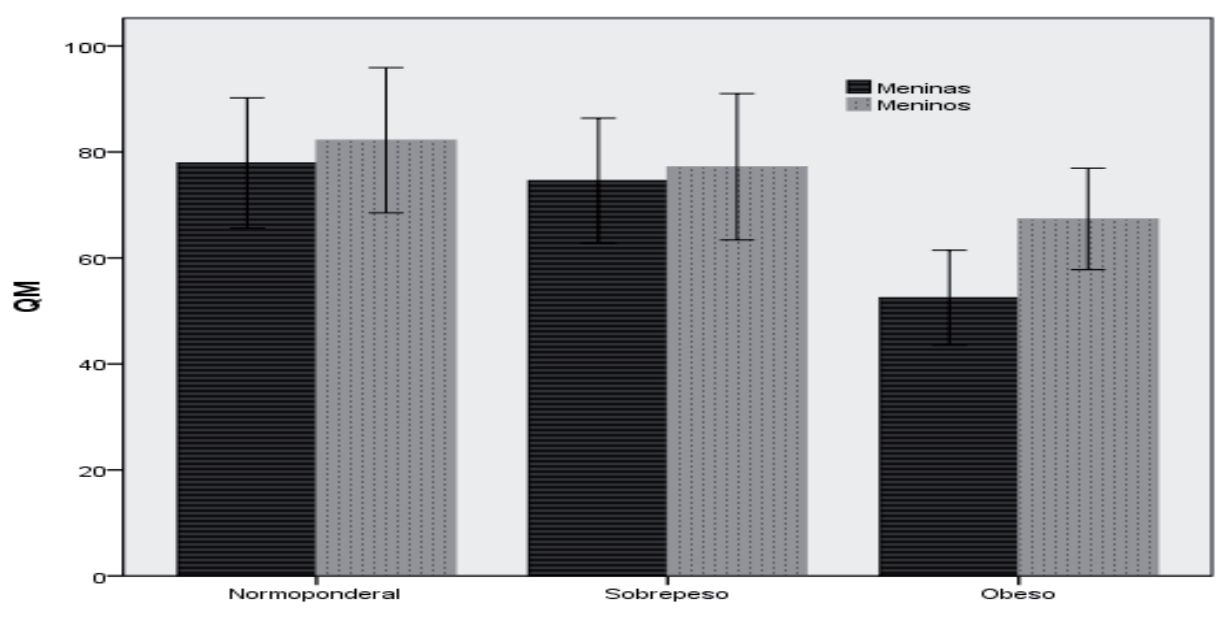

FIGURA 1 - Níveis de coordenação motora (QM) (média \pm desvio-padrão) por categorias do IMC nas meninas e nos meninos. 
TABELA 4 - Valores do coeficiente de correlação de Spearman entre o IMC e o QM nos meninos e nas meninas por idade.

\begin{tabular}{ccc}
\hline Idade (anos) & Meninas & Meninos \\
\hline 6 & $-0,31^{*}$ & $-0,23$ \\
7 & $-0,36^{* *}$ & $-0,23^{*}$ \\
8 & $-0,40^{* *}$ & $-0,39^{* *}$ \\
9 & $-0,50^{* *}$ & $-0,16$ \\
\hline
\end{tabular}

*Significativo para $p<$ 0,05 .

** Significativo para $p$ $<0,01$.

\section{Discussão}

O propósito deste estudo foi o de estudar a associação entre o IMC e a coordenação motora avaliada através da bateria KTK ${ }^{19}$. De acordo com os valores de corte do IOTF para o IMC ${ }^{18}$, a prevalência de sobrepeso e de obesidade é de 20 e 9,8\% respetivamente, o que é consistente com os dados para população infantil portuguesa da mesma idade, sendo $31,5 \%$ obesas ou com sobrepeso ${ }^{3}$.

Uma das principais causas da obesidade, quer em crianças quer em adultos, é o sedentarismo. As crianças despendem muito tempo vendo televisão ou em outras atividades sedentárias em frente a uma tela ${ }^{20-21}$.

Embora um largo número de crianças não cumpra as recomendações dos níveis de $\mathrm{AF}^{22}$, acredita-se que as crianças são por natureza ativas, e envolvemse com frequência em atividades lúdicas ativas desde que se lhe dê oportunidade ${ }^{23}$. Foi sugerido que a principal fonte de motivação para a atividade ou para o jogo ativo está intrinsecamente ligada ao prazer associado com o movimento e a sensação de competência motora ${ }^{15}$. A coordenação motora pode assim ser considerada um agente importante no aumento dos níveis de AF e, por consequência, na redução dos valores obesidade nas crianças.

Os resultados do presente estudo mostram que são as crianças com sobrepeso e obesas, aquelas que marcadamente apresentam baixos níveis de coordenação motora, comparativamente às crianças com peso normal, que o IMC e a coordenação motora estão negativamente correlacionados e que as correlações são baixas a moderadas. Outros estudos encontraram resultados idênticos ${ }^{24}$. Num estudo longitudinal ${ }^{25}$ verificou-se que a coordenação motora era um preditor significativo das mudanças no IMC das crianças. O presente estudo tem um delineamento transversal, pelo que não é possível retirar inferências de causalidade da associação entre o IMC e a coordenação motora.

Se a coordenação motora atuar como promotor da $\mathrm{AF}$ nas crianças, a promoção da $\mathrm{AF}$ na infância deve enfatizar o desenvolvimento da competência motora. Este é um aspecto da maior importância para a educação física curricular nas escolas do ensino primário.

No contexto do estudo das variáveis associadas aos níveis de $\mathrm{AF}$ das crianças algumas pesquisas debruçaram-se sobre a importância da proficiência motora, isto é, sobre a importância na competência motora em habilidades motoras, na coordenação e na aptidão física. OKELY et al. ${ }^{12}$ analisaram a relação entre a competência motora em habilidades motoras básicas e a $\mathrm{AF}$ não organizada e a $\mathrm{AF}$ organizada, tendo verificado que as habilidades motoras básicas explicam 3\% da variância total da AF organizada. Não encontraram associação entre as habilidades motoras básicas e a $\mathrm{AF}$ não organizada. De fato, tal como os resultados encontrados no presente estudo a literatura disponível apresenta uma associação baixa entre a $\mathrm{AF}$ e a proficiência em habilidades motoras ${ }^{13,26}$. Ainda assim, e comparativamente com a aptidão física e a composição corporal, a competência motora é o fator que mais se parece associar com a AF de crianças em idade escolar $(r=0,21$ a 0,55$)$, e pré-escolar $(\mathrm{r}=0,18)^{13,14,15,27}$. A somar a tudo isto, LOPES et al..$^{28}$ verificaram, num estudo longitudinal, em crianças seguidas entre os seis e os 10 anos de idade que a coordenação motora foi o preditor mais importante dos níveis de AF. De fato, as crianças de ambos os sexos que aos seis anos se encontravam no tercil superior da distribuição dos níveis coordenativos não apresentaram declínio dos níveis de $\mathrm{AF}$, enquanto nas restantes houve um decréscimo acentuado.

Os resultados do presente estudo mostram um aumento da correlação entre o IMC e a coordenação motora entre os seis e os oito anos de idade em ambos os sexos e entre os seis e os nove anos nas meninas. Em termos genéricos os resultados do presente estudo estão em consonância com o modelo preditivo proposto por STODDEN et al. ${ }^{29}$. Durante a infância existe uma clara tendência 
para a associação entre o IMC e a coordenação motora aumentar, fruto, provavelmente, dos efeitos bidireccionais entre as duas variáveis. A criança com bom nível de coordenação motora tende a ter sucesso nas atividades físicas pelo que estará mais motivada para continuar a participar nelas, reforçando a sua competência percebida e a aptidão física e, consequentemente, mantendo uma composição corporal mais adequada (IMC menor). O oposto é expectável em crianças com baixo nível de coordenação motora. Este hipotético "feedback" reforça a relação entre o IMC e a coordenação motora verificada no aumento dos valores de correlação com a idade durante a infância, observados no presente estudo.
Em conclusão, a coordenação motora está moderada e negativamente associada com o IMC e a associação aumenta durante a infância. As crianças com sobrepeso e obesas de ambos os sexos apresentam menores níveis de coordenação motora do que as crianças normoponderais. Apesar de o presente estudo ter um delineamento transversal, a coordenação motora parece ser um fator importante nos valores do IMC.

Os resultados deste estudo destacam a potencial importância da promoção do desenvolvimento da coordenação motora nas crianças na diminuição dos níveis de obesidade. A instrução sistemática e a disponibilidade de tempo e espaço adequados para a prática são fatores significativos que contribuem para a melhoria da proficiência motora nas crianças ${ }^{30}$.

\begin{abstract}
The association between body mass index and motor coordination in children

The purpose of this study was to analyze the association between body mass index (BMI) and motor coordination (MC) in children. Data were collected in 794 children, ages 6-9 years. BMI was calculated from measured height and weight [Body mass $(\mathrm{kg}) /$ Height $\left.\left(\mathrm{m}^{2}\right)\right]$. MC was evaluated using KTK. In both girls $\left(\chi^{2}\right.$ $=93.96 ; p<0.001)$ and boys $\left(\chi^{2}=46.98 ; p<0.001\right)$. There were significant differences in MC between the three groups of BMI (normal weight, overweight, obese). Normal weight children of both sexes have better outcomes than those who are overweight, and these in turn have better results than the obese. MC is moderately and negatively associated with $\mathrm{BMI}$ and the association increases during childhood $(-0.16$ to -0.50 ). In both boys and girls overweight and obese have lower levels of MC than normal weight children.
\end{abstract}

KEY WORDS: Obesity; Psychomotor performance; Physical activity.

\title{
Referências
}

1. World Health Organization (WHO). Obesity and overweight. Geneva: WHO; 2006.

2. World Health Organization (WHO). Prevalence of excess body weight and obesity in children and adolescents.Geneva: WHO; 2007.

3. Padez C, Fernandes T, Mourão I, Moreira P, Rosado V. Prevalence of overweight and obesity in 7-9 year old portuguese children: Trends in body mass index from 1970-2002. Am J Hum Biol. 2004;16:670-8.

4. Wu DM, Hong Y, Sun CA, Sung PK, Rao DC, Chu NF. Familial resemblance of adiposity-related parameters: Results from a health check-up population in taiwan. Eur J Epidemiol. 2003;18:221-6.5.

5. Baba R, Koketsu M, Nagashima M, Inasaka H. Role of exercise in the prevention of obesity and hemodynamic abnormalities in adolescents. Pediatr Int. 2009;51:359-63.

6. Reilly JJ. Low levels of objectively measured physical activity in preschoolers in child care. Med Sci Sports Exerc. 2010; 42:502-7.

7. Malina RM, Bouchard C, Bar-Or O. Growth, maturation and physical activiy. Champaign: Human Kinetics; 2004.

8. Sallis JF, Prochaska JJ, Taylor WC. A review of correlates of physical activity of children and adolescents. Med Sci Sports Exerc. 2000;32:963-75.

9. Horst KVD, Paw MJCA, Twisk JWR, Mechelen WV. A brief review on correlates of physical activity and sedentariness in youth. Med Sci Sports Exerc. 2007;39:1241-50. 
10. Sallis JF, Alcaraz JE, McKenzie TL, Hovell MF. Predictors of change in children's physical activity over 20 months. Variations by gender and level of adiposity. Am J Prev Med. 1999;16:222-9.

11. Butcher JE, Eaton WO. Gross and fine motor proficiency in pre-schoolers: Relationships with free play behaviour and activity level. J Hum Mov Studies. 1989;16:27-36.

12. Okely AD, Booth ML, Patterson JW. Relationship of physical activity to fundamental movement skills among adolescents. Med Sci Sports Exerc. 2001;33:1899-904.

13. Fisher A, Reilly JJ, Kelly LA, et al. Fundamental movement skills and habitual physical activity in young children. Med Sci Sports Exerc. 2005;37:684-99.

14. Raudsepp L, Päll P. The relationship between fundamental motor skills and outside-school physical activity of elementary school children. Pediatr Exerc Sci. 2006;18:426-35.

15. Wrotniak BH, Epstein LH, Dorn JM, Jones KE, Kondilis VA. The relationship between motor proficiency and physical activity in children. Pediatrics. 2006;118:e1758-65.

16. Williams HG, Pfeiffer KA, O’Neill JR, et al. Motor skill performance and physical activity in preschool children. Obesity. 2008;16:1421-6.

17. Rose B, Larkin D, Berger BG. The importance of motor coordination for children's motivational orientations in sport. Adapt Phys Activ Q. 1998;15:316-27.

18. Cole TJ, Bellizzi MC, Flegal KM, Dietz WH. Establishing a standard definition for child overweight and obesity worldwide: international survey. BMJ. 2000;320:1240-3.

19. Kiphard EJ, Schilling F. Korper-koordinations-test fur kinder. Ktk. Manual. Weiheim: Beltz Test GmbH; 1974.

20. Jago R, Baranowski T, Baranowski JC, Thompson D, Greaves KA. Bmi from 3-6 y of age is predicted by tv viewing and physical activity, not diet. Int J Obes. 2005;29:557-64.

21. Nelson MC, Neumark-Stzainer D, Hannan PJ, Sirard JR, Story M. Longitudinal and secular trends in physical activity and sedentary behavior during adolescence. Pediatrics. 2006;118:e1627-34.

22. Nilsson A, Anderssen SA, Andersen LB, et al. Between- and within-day variability in physical activity and inactivity in 9- and 15-year-old european children. Scand J Med Sci Sports. 2009;19:10-8.

23. Burdette HL, Whitaker RC. Resurrecting free play in young children: Looking beyond fitness and fatness to attention, affiliation, and affect. Arch Pediatr Adolesc Med. 2005;159:46-50.

24. Graf C, Koch B, Kretschmann-Kandel E, et al. Correlation between bmi, leisure habits and motor abilities in childhood (chilt-project). Int J Obes Relat Metab Disord. 2004;28:22-6.

25. Martins D, Maia J, Seabra A, et al. Correlates of changes in BMI of children from the Azores islands. Int J Obes. 2010;34:1487-93.

26. Cliff DP, Okely AD, Smith LM, McKeen K. Relationships between fundamental movement skills and objectively measured physical activity in preschool children. Pediatr Exerc Sci. 2009;21:436-49.

27. D'Hondt E, Deforche B, Bourdeaudhuij ID, Lenoir M. Relationship between motor skill and body mas index in 5- to 10-year-old children. Adapt Phys Activ Q. 2009;26:21-37.

28. Lopes VP, Rodrigues LP, Maia JAR, Malina RM. Motor coordination as predictor of physical activity in childhood. Scand J Med Sci Sports. 2011;21:663-9.

29. Stodden DF, Goodway JD, Langendorfer SJ, et al. A developmental perspective on the role of motor skill competence in physical activity: an emergent relationship. Quest. 2008;60:290-306.

30. Malina RM, Little BB. Physical activity: the present in the context of the past. Am J Hum Biol. 2008;20:373-91.

\begin{tabular}{r|l} 
ENDEREÇo & \\
Maria Mafalda Melo & \\
Universidade Estadual Paulista - Campus Rio Claro & Recebido para publicação: 20/12/2011 \\
Av. Doutor Morato, 967 & Revisão: 28/03/2012 \\
13405-260 - Piracicaba - SP - BRASIL & Aceito: 18/07/2012 \\
e-mail: mafaldaportugal@gmail.com & \\
&
\end{tabular}

